\title{
Influence of misalignments on the performance of externally occulted solar coronagraphs \\ Application to PROBA-3/ASPIICS
}

\author{
S. V. Shestov ${ }^{1,2}$ and A. N. Zhukov ${ }^{1,3}$ \\ ${ }^{1}$ Solar-Terrestrial Centre of Excellence - SIDC, Royal Observatory of Belgium, Avenue Circulaire 3, 1180 Brussels, Belgium \\ e-mail: s.shestov@oma.be \\ ${ }^{2}$ Lebedev Physical Institute, Leninskii prospekt, 53, 119991 Moscow, Russia \\ ${ }^{3}$ Skobeltsyn Institute of Nuclear Physics, Moscow State University, Leninskie gory, 119991 Moscow, Russia
}

Received 29 November 2017 / Accepted 11 January 2018

\begin{abstract}
Context. The ASPIICS instrument is a novel externally occulted coronagraph that will be launched on board the PROBA-3 mission of the European Space Agency. The external occulter will be placed on one satellite $\sim 150 \mathrm{~m}$ ahead of the second satellite that will carry an optical instrument. During $6 \mathrm{~h}$ out of $19.38 \mathrm{~h}$ of orbit, the satellites will fly in a precise (accuracy around a few millimeters) formation, constituting a giant externally occulted coronagraph. The large distance between the external occulter and the primary objective will allow observations of the white-light solar corona starting from extremely low heights $\sim 1.1 R_{\odot}$.

Aims. We intend to analyze influence of shifts of the satellites and misalignments of optical elements on the ASPIICS performance in terms of diffracted light. Based on the quantitative influence of misalignments on diffracted light, we provide a recipe for choosing the size of the internal occulter (IO) to achieve a trade-off between the minimal height of observations and sustainability to possible misalignments.

Methods. We considered different types of misalignments and analyzed their influence from optical and computational points of view. We implemented a numerical model of the diffracted light and its propagation through the optical system and computed intensities of diffracted light throughout the instrument. Our numerical approach is based on a model from the literature that considered the axisymmetrical case. Here we extend the model to include nonsymmetrical cases and possible misalignments.

Results. The numerical computations fully confirm the main properties of the diffracted light that we obtained from semi-analytical consideration. We obtain that relative influences of various misalignments are significantly different. We show that the internal occulter with $R_{I O}=1.694 \mathrm{~mm}=1.1 R_{\odot}$ is large enough to compensate possible misalignments expected to occur in PROBA-3/ASPIICS. Besides that we show that apodizing the edge of the internal occulter leads to additional suppression of the diffracted light.

Conclusions. We conclude that the most important misalignment is the tilt of the telescope with respect to the line connecting the center of the external occulter and the entrance aperture. Special care should be taken to co-align the external occulter and the coronagraph, which means co-aligning the diffraction fringe from the external occulter and the internal occulter. We suggest that the best orientation strategy is to point the coronagraph to the center of the external occulter.
\end{abstract}

Key words. Sun: corona - instrumentation: high angular resolution - telescopes - methods: numerical

\section{Introduction}

The solar corona is the outer layer of the solar atmosphere. This outer layer consists of highly ionized plasma that is structured by the magnetic field. In the solar corona a number of important but still ill-understood phenomena take place, such as the initiation and acceleration of coronal mass ejections (CMEs) and the acceleration of the solar wind.

Currently the corona is observed by space-borne telescopes and coronagraphs in various spectral ranges from X-rays and extreme ultraviolet (EUV) to white light. The EUV spectral range is more suitable for observations of the low corona due to the extremely low brightness of plasma in EUV at high altitudes that decreases with height proportionally to the square of the electron density $n_{e}^{2}$, contrary to white-light brightness proportional to $n_{e}$. There are occasional observations up to $2 R_{\odot}$ with regular EUV telescopes that operated in dedicated long-exposure regimes, e.g., TESIS/CORONAS-PHOTON (Kuzin et al. 2011;
Reva et al. 2014) or SWAP/PROBA-2 (Berghmans et al. 2006; Seaton et al. 2013; D'Huys et al. 2017), or up to $2.5 R_{\odot}$ with specialized EUV coronagraphs, e.g., SPIRIT/CORONAS-F (Zhitnik et al. 2003; Slemzin et al. 2008).

White-light coronagraphs can provide reliable observations over a significantly larger field of view due to a different mechanisms of emission, primarily Thomson scattering. For example, the field of view of the internally occulted coronagraph COR1/STEREO ranges from 1.5 to $4.0 R_{\odot}$ (Howard et al. 2008). The field of view of externally occulted coronagraphs usually starts slightly higher, from $\sim 2.2 R_{\odot}$ in LASCO C $2 / \mathrm{SOHO}$ (Brueckner et al. 1995), and from $2.5 R_{\odot}$ in COR2/STEREO-A (Howard et al. 2008; see also comparison of various coronagraphs in Frazin et al. 2012). The very important range of heights from $1.1 R_{\odot}$ to $2.5 R_{\odot}$ (Zhukov et al. 2008 ) is almost not covered in white-light observations from space, except for LASCO $\mathrm{C} 1 / \mathrm{SOHO} \sim 1.1-3.0 R_{\odot}$ (Brueckner et al. 1995), which suffered from significant stray light. 
Classical internally occulted coronagraphs cannot provide such observations because the theoretical limit of diffracted light around $10^{-4} B_{\odot}$ at $1.1 R_{\odot}$ exceeds the brightness of the solar corona $10^{-5} B_{\odot}$ (see review of various coronagraph systems in Rougeot et al. 2017, hereafter RR17). Scattering of the bright light from the solar disk in the primary objective further worsens the problem. The intensity of diffracted light in the inner corona region can be decreased with the use of an apodized entrance aperture (Aime 2007), but this solution has not yet been implemented in a solar coronagraph.

For externally occulted coronagraphs, there are two fundamental factors that complicate observations at low heights: the drastic increase of diffracted light and the vignetting of the inner field of view (see, e.g., Koutchmy 1988; Kim et al. 2017). Furthermore, straightforward ways to compensate both effects are contradictory; diffracted light decreases (Lenskii 1988; Fort et al. 1978), whereas vignetting increases with the increase of the external occulter size (or putting it closer to the primary objective). In all the previous space-borne coronagraphs, the distance from the external occulter to the primary objective was limited by the length of the instrument, i.e., $\sim 1 \mathrm{~m}$. This fact does not allow the use of sufficiently large primary objectives and simultaneously results in almost $50 \%$ vignetting of the objective throughout the field of view.

The ASPIICS (Association of Spacecraft for Polarimetric and Imaging Investigation of the Corona of the Sun) instrument is a novel white-light coronagraph (Lamy et al. 2010; Renotte et al. 2015, 2016) that will perform regular observations of the corona over the field of view from $\sim 1.08 R_{\odot}$ up to $3.0 R_{\odot}$. This will be possible thanks to European Space Agency PROBA-3 mission that will use formation flying (FF). Such a technology allows virtual enlarging of instrumentation to an unprecedented size; the external occulter will be placed on one satellite and the optical instrument on the second. Both satellites will synchronously fly on a highly elliptical $19.38 \mathrm{~h}$ orbit and form a precise formation with inter-satellite distance $\sim 150 \mathrm{~m}$ during 6 hours. The external occulter with $\varnothing 1.42 \mathrm{~m}$ diameter will produce a $\emptyset 77 \mathrm{~mm}$ shadow and the telescope with the entrance aperture $\oslash 50 \mathrm{~mm}$ will be placed in the center with the maximal possible precision. The giant inter-satellite distance allows unvignetted observations of the solar corona already starting from $\approx 1.1 R_{\odot}$.

In order to provide highest possible accuracy of FF, the satellites are equipped with fine metrology subsystems that include laser beam/reflector and shadow position sensor (SPS; Renotte et al. 2016; Bemporad et al. 2015). The SPS consists of eight photodiodes placed around the entrance aperture of the telescope in the penumbra region. The SPS will measure relative position of the satellites with the accuracy better than $\sim 1 \mathrm{~mm}$ in longitudinal and transversal directions. The expected FF accuracies are $\pm 15 \mathrm{~mm}$ in longitudinal and $\pm 5 \mathrm{~mm}$ in transversal $x$ - and $y$ directions, 30 arcmin for the attitude of the occulter satellite, and 15 arcsec for the attitude of the satellite with the telescope ( $3 \sigma$ values).

Various analyses (Landini et al. 2010; Aime 2013) show that the amount of diffracted light reaching the ASPIICS primary objective is high enough. In order to reduce its level, the external occulter of ASPIICS will have a toroidal shape; technological limitations do not allow the use of a superior external occulter such as a multi-disk system or a threaded-cone (see Bout et al. 2000, for review of various systems). The RR17 work used an analytical and numerical approach and calculated not only the diffraction level just in front of the primary objective, but, more importantly, the spatial distribution of diffracted light
Table 1. Key planes of the ASPIICS coronagraph.

\begin{tabular}{ll}
\hline \hline Plane & Description \\
\hline$O$ & External occulter plane \\
$A$ & Entrance aperture of the telescope \\
$B$ & Focal plane of the primary objective \\
$O^{\prime}$ & Conjugate image of plane $O$ (plane of the IO) \\
$C$ & Conjugate image of plane $A$ (plane of the Lyot stop) \\
$D$ & Final focal plane and the detector \\
\hline
\end{tabular}

at the detector. The authors found that the intensity drastically depends on the sizes of the internal occulter and the Lyot stop, and provided a recipe for choosing their proper sizes. The analysis, however, was only limited to the symmetrical case, when the Sun, the external occulter, and the coronagraph are on the same axis and are perfectly co-aligned.

Besides the misalignments introduced by FF, there are additional sources of misalignments expected in ASPIICS. These can be long- or short-term thermal expansions of mechanical structure, errors of initial co-alignment of the telescope on the satellite, etc. In total these misalignments can be very small, on the order of 10-30 arcsec. Nevertheless, as Venet et al. (2010) concluded, even such low values can be critical for coronagraphs with extremely small overoccultation.

The aim of the present paper is to investigate how different misalignments influence the intensity of the diffracted light in the final image, to compare this intensity with that of the corona and to choose the proper size of the internal occulter to ensure reliable rejection for the cases of possible misalignments. The analysis considers only the effect of diffraction, i.e., the effect that is caused by masking of individual parts of the wavefront of propagating light. Other effects that are unavoidably present and degrade the performance of the coronagraph, such as scattered light and ghosts, and non-ideal lenses, provide an additional contribution but are not considered here. Whereas the computations are performed for a particular geometry and optical layout (representative for the ASPICS coronagraph), we note that the obtained properties of the diffracted light and the behavior of diffracted light with various misalignments remain valid for any externally occulted coronagraph.

The paper is structured as follows: in Sect. 2 we describe the optical layout and the basics of the algorithm, in Sect. 3 we discuss some properties of the diffracted light, and in Sect. 4 we consider possible types of misalignments. In Sect. 5 we present results of computations and consider different sizes of internal occulter (IO) and its apodization. In Sect. 6 we discuss the obtained results and give conclusions in Sect. 7. Appendix A contains mathematical details of the calculations, Appendix B details the numerical computations, and Appendix $\mathrm{C}$ analyzes influence of samplings.

\section{Optical layout, model, algorithm}

\subsection{Optical layout}

A detailed description of the optical layout of ASPIICS is given by Galy et al. (2015). In the present paper we use a simplified model and conventions to denote optical planes described in RR17. Table 1 summarizes the names and description of the optical planes. 


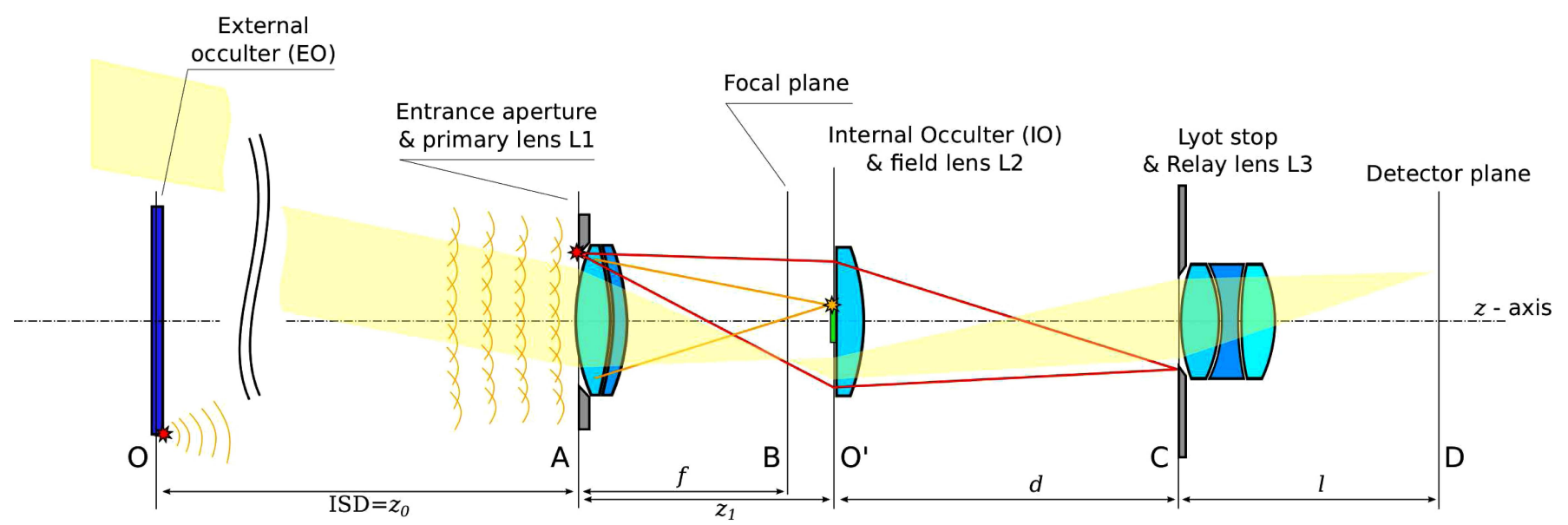

Fig. 1. Optical layout of the ASPIICS coronagraph. The dim yellow light represents a regular coronal beam, the orange arches and lines represent the light that diffracts on the EO, and the red lines represent the light diffracted at the entrance aperture.

The optical layout of the ASPIICS coronagraph is given in Fig. 1. The external occulter (EO; plane $O$ ) is situated on the occulter satellite placed at the distance $z_{0}=144348 \mathrm{~mm}$ ahead of the coronagraph satellite with the optical instrument. The telescope consists of the entrance aperture (plane $A$ ) and the primary lens L1 with the focal length $f$, which makes an image of the corona in the primary focal plane $B$. The light diffracted at the EO is focused on the $O^{\prime}$ plane, situated at the distance $z_{1}$. This is the plane of the conjugate image of the EO produced by L1, thus it is $\Delta=z_{1}-f \approx 0.76 \mathrm{~mm}$ further than $B$. The major part of the diffracted light is cut by the internal occulter, the size of which should be carefully chosen to obtain a good rejection of diffraction and not to vignette too much. The IO is deposited directly on the surface of the field lens L2. The lens L2 along with L1 make an image of the entrance aperture on the $C$ plane. The Lyot stop is placed in this plane and rejects the light diffracted at the entrance aperture. Simultaneously, the lens L2 projects the entrance aperture to the relay lens L3, ensuring that the light propagates further into the coronagraph and justifying its name - the field lens. Finally, both L2 and L3 project the primary focus $B$ onto the detector plane $D$.

In Fig. 1 the dim yellow light represents a regular coronal beam and the orange arches represent light that diffracts on the EO. After propagating through the entrance aperture and L1, this light is focused (orange lines) in the $O^{\prime}$ plane. The red lines represent the light diffracted at the entrance aperture.

Our optical model contains two main simplifications: we place the entrance aperture and the primary objective on the same plane $A$ (in the real instrument the objective is $\sim 120 \mathrm{~mm}$ behind), and we change the distances $d, l$, and the focal distance of L3 to keep the same spatial scale in $D$ as in $B$. Neither simplification influences the diffracted light.

We perform the calculation for the central wavelength $550 \mathrm{~nm}$ of the main (wideband) passband and discuss possible effects in Sect. 6.3.

Table 2 summarizes main parameters of the ASPIICS optical layout and other parameters used in our computations.

We consider an infinitely thin (razor edge) external occulter. In reality the edge has a toroidal cross-section both for improvement of the diffracted light rejection (Landini et al. 2010) and for reduction of the effect of tilting. The simplification used here is linked to the difficulty of a correct representation of 3D occulters in the framework of the Fresnel diffraction (Sirbu et al. 2016). Currently attempts are ongoing to take the effect of the
Table 2. Parameters used in our numerical study taken from the ASPIICS configuration.

\begin{tabular}{lll}
\hline \hline Parameter & Symb. & Value \\
\hline Wavelength & $\lambda$ & $550 \mathrm{~nm}$ \\
Angular radius of the Sun & $R_{\odot}$ & $16 \mathrm{arcmin}$ \\
Radius of the EO & $R_{\mathrm{EO}}$ & $710 \mathrm{~mm}$ \\
Distance plane $O$ - plane $A$ & $z_{0}$ & $144348 \mathrm{~mm}$ \\
Angular radius of the EO & $\omega_{\mathrm{EO}}$ & $16.909 \mathrm{arcmin}$ \\
Radius of the entrance aperture & $R_{A}$ & $25.0 \mathrm{~mm}$ \\
Focal length of L1 & $f$ & $330.348 \mathrm{~mm}$ \\
Distance plane $A-$ plane $O^{\prime}$ & $z_{1}$ & $331.143 \mathrm{~mm}$ \\
Radius of the internal occulter & $R_{\mathrm{IO}}$ & $1.662 \mathrm{~mm}^{a}$ \\
Hole in the internal occulter & $r_{\mathrm{IO}}$ & $0.489 \mathrm{~mm}^{b}$ \\
Lyot stop radius & $R_{C}$ & $0.97 R_{A}(24.25 \mathrm{~mm})^{c}$ \\
\hline
\end{tabular}

Notes. See text for details. ${ }^{(a)}$ The value that we use as a baseline for the diffracted light. ${ }^{(b)}$ A hole in the internal occulter is used to produce images of LEDs from the occulter satellite on the detector. ${ }^{(c)}$ The relative size of the entrance aperture and the Lyot stop plays a role.

toroidal shape of the occulter into account both experimentally and numerically.

\subsection{Projection of the Sun, EO, and IO onto different planes}

It is interesting to compare how different objects, that is, the Sun, $\mathrm{EO}$, and IO, are projected in the framework of the geometrical optics onto planes $B$ and $O^{\prime}$. It turns out that the geometrical approach correctly predicts the relative sizes of images and their relative shifts owing to the tilt of the coronagraph or other misalignments. Since $O$ and $O^{\prime}$ are conjugate planes, we can project the IO onto $O$ and after that onto $B$. In the figures below we show the EO and IO with a transparent central part for clarity.

We start with the case of the perfect symmetry, when the coronagraph and the EO are co-aligned and co-centered, and the center of the Sun lies on the same optical axis $z$. In Fig. 2 we present sketches with plane $B$ in the left panel and plane $O^{\prime}$ in the right panel.

The Sun is sharply focused on the $B$ plane and is slightly defocused on the $O^{\prime}$ plane; we denote defocusing by a smooth limb. 

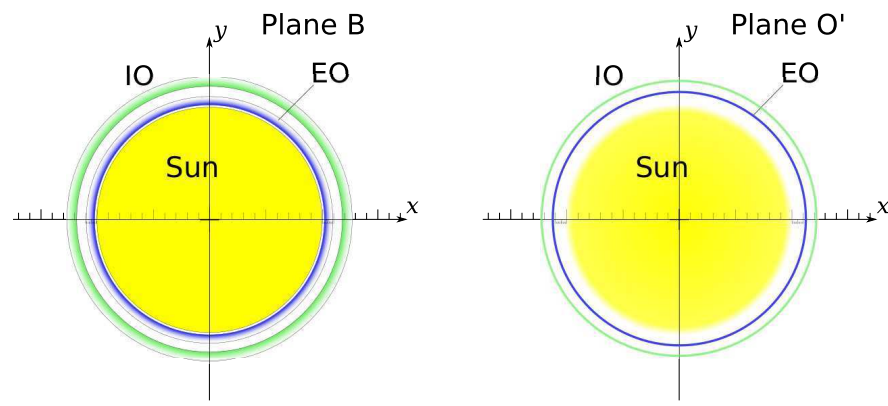

Fig. 2. Projections of the Sun, the EO and IO onto planes $B$ and $O^{\prime}$ in the symmetrical case.

The EO is represented in blue in both planes in Fig. 2. In the $O^{\prime}$ plane the image of EO is perfectly focused, which is denoted by a thin ring. In the $B$ plane the image of the EO is defocused, thus the EO is indicated as a ring with a finite thickness and gradient filling. Inner and outer edges of the ring correspond to the extremes in optical vignetting produced by the EO, and the gradient itself represents the vignetting function (full obscuration inside and full transparency outside). Obviously, the angular size of the EO should be selected in such a way that its inner vignetting zone is larger than the Sun.

The IO is represented by a green ring. Similar to the EO, the IO is perfectly focused on the $O^{\prime}$ plane and defocused on the $B$ plane. The reasoning regarding the inner and outer edges can be applied here as well. The size of the IO should be selected in such a way that it fully covers both the Sun and EO.

\subsection{Algorithm of the diffraction calculation}

In our analysis we follow the basic idea of Aime (2013) and RR17. Here we briefly summarize the method, and we give the full mathematical description in Appendix A.

We set up a reference frame with the $x y$ plane coinciding with the $O$ plane and co-centered with the EO and the $z$-axis pointing to the entrance aperture; this implies the co-alignment and co-centering of the coronagraph and the EO. The Sun is considered as a spatially extended light source that produces a set of noncoherent plane-parallel waves with wave-vectors $\boldsymbol{k}$, i.e.,

$\Psi_{\boldsymbol{k}}=A \exp (-i(\omega t-\boldsymbol{k r}))$

By varying the direction of $\boldsymbol{k}$ we sample the solar disk (so far it needs not to be co-aligned).

The algorithm for calculating the diffracted light in various planes consists of the following:

1. We select a particular point on the Sun and considered its plane-parallel wave $\Psi_{\boldsymbol{k}}$. The direction of the wave-vector $\boldsymbol{k}$ can be specified in the $B$ plane either by Cartesian $(\alpha, \beta)$ or by polar $(\rho, \varphi)$ coordinates. The intensity of the wave depends on the solar brightness and sampling area $A^{2}=$ $B_{k} \mathrm{~d} S$.

2. After diffraction at the EO, each wave becomes significantly not a plane-parallel wave, and it cannot be expressed similarly to (1). We express its amplitude in the $A$ plane as $\Psi_{A k}$, where $\boldsymbol{k}$ denotes the orientation of initial wave.

3. Further propagation of the wave is considered in the framework of the Fourier optics formalism. The fields in the $O^{\prime}, C$, and $D$ planes are calculated with three successive FourierFresnel transforms over the distances $z_{1}, d, l$ and taking into account apertures $A_{A}, A_{O^{\prime}}$, and $A_{C}$.
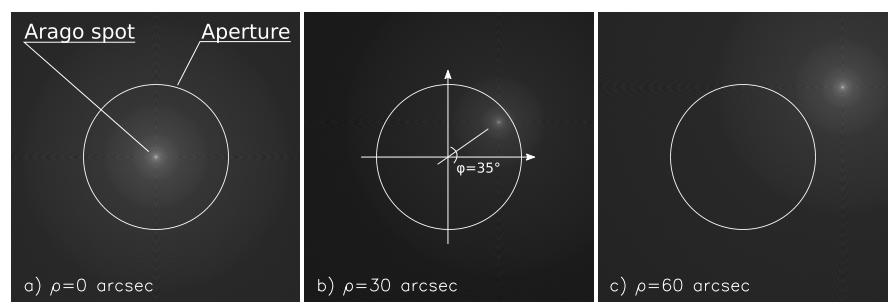

Fig. 3. Intensities $\left|\Psi_{A k}\right|^{2}$ of waves in the $A$ plane propagating with different directions of $\boldsymbol{k}_{\rho \varphi}:(a) \rho=0,(b) \rho=30^{\prime \prime}$, and $(c) \rho=60^{\prime \prime}\left(\varphi=35^{\circ}\right.$ in the latter two cases). Only the part of $\Psi_{A}$ that enters into the aperture produces signal in further planes of the telescope.

4. We consider all the possible waves, summarizing over directions of $\boldsymbol{k}$ (i.e., over $(\rho, \varphi)$ or $(\alpha, \beta)$ ), and calculate the final image in $D$ as

$$
I_{D}=\sum_{k} B_{k}\left|\Psi_{D k}\right|^{2} \mathrm{~d} S
$$

We stress that the $\boldsymbol{k}$ vector is determined in the reference frame of the EO and coronagraph, and not that of the Sun. All the characteristics of the Sun - the solar brightness, angular size, and limb darkening - are implicitly hidden in $B_{\boldsymbol{k}}$ and possible $\boldsymbol{k}$ directions, whereas the obscuration by the EO and IO is implicitly hidden in $\Psi$.

Calculation of $\Psi_{A k}$ is a separate problem as it involves computing of the Fresnel diffraction on the EO. Again, we follow approach of Aime (2013) and RR17, who considered an infinitely thin (razor edge) external occulter. The details are given in Appendix A, and we note that $\Psi_{A \boldsymbol{k}}$ for a particular $\boldsymbol{k}$ can be calculated from the coaxial wave $\Psi_{A 00}(\rho=0, \varphi=0)$ by shifting and multiplicating by an arbitrary phase function. We give several examples of $\Psi_{A k}$ in Fig. 3, where the three panels correspond to different $\boldsymbol{k}$ : the coaxial $\boldsymbol{k}$ with $\rho=0$ (this is essentially $\Psi_{A 00}$ ), and two tilted $\boldsymbol{k}$ with $\rho=30^{\prime \prime}$ and $\rho=60^{\prime \prime}\left(\varphi=35^{\circ}\right.$ in the latter two cases). The central bright feature of the coaxial wave, that is, the Arago spot, is displaced in tilted waves. The highly oscillating nature of the $\Psi_{A k}$ function (see Fig. 2 in RR17) is present but is not visible here in the pdf and hardcopy images. Only the part of the wave that fits the entrance aperture produces further signal in the instrument. The full-Sun umbra-penumbra pattern in the aperture plane can be obtained by integrating $\Psi_{A k}$ over the Sun and resembles geometrical umbra-penumbra pattern with some signal in the umbra region (see Fig. 5 in RR17).

\section{Some properties of diffraction}

Calculation of the diffracted image in the $O^{\prime}$ plane $I_{O^{\prime}}=$ $\sum_{k} B_{k}\left|\Psi_{O^{\prime} k}\right|^{2} \mathrm{~d} S$ reveals that it is a bright ring with radius $r=$ $z_{1} \tan \left(\omega_{E O}\right)$, that is, with the angular size exactly coinciding with the angular size of the $\mathrm{EO}-\omega_{\mathrm{EO}}=\arctan \left(R_{\mathrm{EO}} / z_{0}\right)$.

One of the main properties of the diffraction is the following: every single wave $\Psi_{A k}$ after propagation into $O^{\prime}$ highlights either a sector or the whole ring (see Fig. 4 with examples of $\left|\Psi_{O^{\prime} k}\right|^{2}$ ). The highlighted sector has the same polar coordinate $\varphi$ as the initial wave, and its angular width depends on $\rho$ of the initial wave: the higher $\rho$, the smaller the width. For the coaxial wave $\rho=0$, and the whole ring is bright (panel a). For the waves with $\rho \approx 10^{\prime}$ and $\rho \approx 16^{\prime}$ the bright sector becomes very narrow (panels $\mathrm{b}$ and $\mathrm{c}$ ). Two-dimensional distributions of intensities have very intense peaks and strongly decreasing wings. 

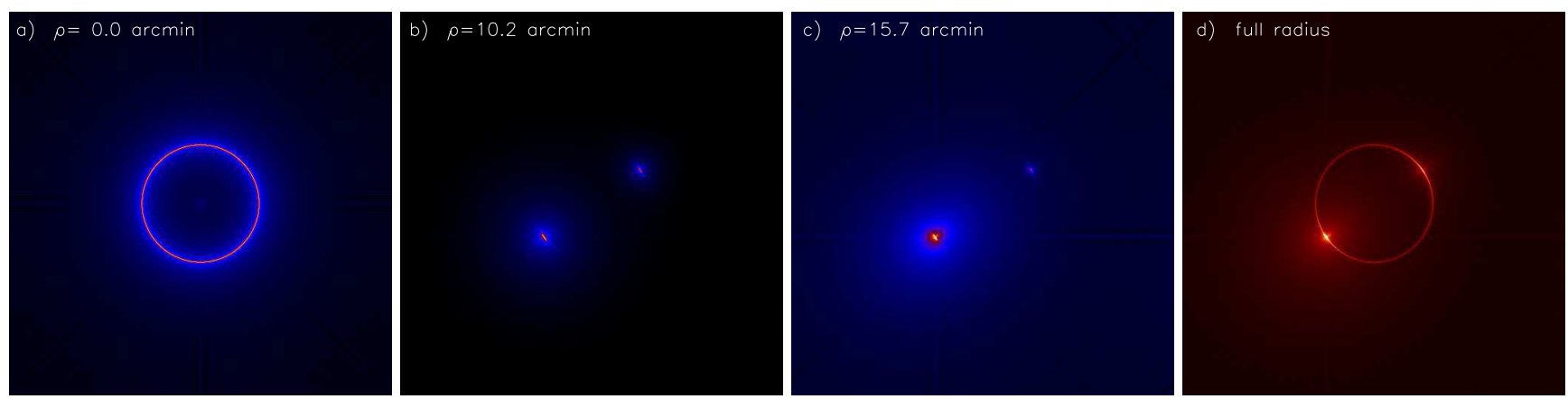

Fig. 4. Intensity of the diffracted wave $\left|\Psi_{O^{\prime} \boldsymbol{k}}\right|^{2}$ in the $O^{\prime}$ plane for different initial waves $\boldsymbol{k}_{\rho \varphi}$. Panel a: A coaxial wave $\rho=0$ is shown; panel $b$ : a moderately tilted wave with $\rho=10.24^{\prime}$ is shown; and panel $c$ : a highly tilted wave with $\rho=16^{\prime}$ is represented. panel $d$ : The intensity is integrated for all the points along the solar radius. The $\mathrm{d} S=\rho \mathrm{d} \rho \mathrm{d} \varphi$ factor was taken into account during integration, thus the color table is different. The value $\varphi=35^{\circ}$ was used in the latter three cases.

This reasoning allows us to infer the second property. If the EO and coronagraph are on the same optical axis, the relative displacement of the Sun from this axis does not change the geometrical symmetry of the diffraction ring in $O^{\prime}$. In the case of such a displacement, individual parts of the diffracted ring become brighter or dimmer, but they do not move in the $O^{\prime}$ plane. Similarly, the seasonal change of the angular size of the Sun does not change the geometry of the diffracted ring in $O^{\prime}$.

The final image in $D$ is formed by diffracted light that propagates behind the IO. As long as the relative shift of the diffracted ring and the IO is small (as expected in ASPIICS), the effect of diffraction determines the final image in $D$. Thus, the third property is the position of the diffraction ring on the detector; the size and shape of the diffraction ring are determined by the IO. Misalignments do not shift or distort the final diffraction ring; they just make individual parts of the ring brighter or dimmer. The variations of the IO size can change the size of the final diffraction ring.

\section{Types of misalignments}

There are various types of misalignments of optical elements that influence the overall intensity and spatial distribution of the diffracted light. Among these are transversal (in the $x y$ plane) shifts of the EO and telescope, tilts of the EO and telescope, shifts and tilts of internal optical elements inside the coronagraph, change of $z_{0}$, etc. The influence of some of the misalignments, such as change of $z_{0}$ or the longitudinal displacement of the IO, can be analyzed using the symmetrical model since they do not break the axial symmetry. Other misalignments, such as the transversal shift of the EO and coronagraph or transversal shifts of apertures, must be considered using a full-sampling approach because the optical layout loses axial symmetry.

Below we show that any configuration with transversal shifts of external optical elements can be reduced to superposition of just two misalignments - the tilt of the telescope and the shift of the Sun.

We do not consider the influence of the possible tilt of the lenses, (the expected tilts on the order of 0.5-5 arcmin are negligible) and the tilt of the EO for the reasons explained in Sect. 2.1.

In the symmetrical case (panel a in Fig. 5) the $z$-axis goes through the center of the Sun, EO, and coronagraph. The $z$-axis is perpendicular to the EO plane and co-aligned with the coronagraph optical axis. The entrance aperture is co-centered with the umbra-penumbra pattern.

In the case the Sun is shifted (panel b in Fig. 5), the EO and the coronagraph remain on the initial $z$-axis. However, the umbra-penumbra pattern is no longer symmetrical with respect to the entrance aperture. The projections of the Sun, EO, and IO onto planes $B$ and $O^{\prime}$ are shown in Fig. 6: the projection of the Sun is shifted, but the EO remains in the same position. The solar shift changes the relative intensity of EO (i.e., the diffraction bright ring), but does not change its geometry or its position because of the property discussed in Sect. 3. The coronagraph and its mathematical model remain axisymmetrical, thus the waves propagating from different directions $\boldsymbol{k}_{\rho \varphi_{1}}$ and $\boldsymbol{k}_{\rho \varphi_{2}}$ produce similar responses in $D$, which are just rotated with respect to each other by the angle $\varphi_{2}-\varphi_{1}$. From the computational point of view the misalignment changes the coordinates, for example, $(\alpha, \beta) \rightarrow(\alpha+\phi, \beta)$, of all the waves $\Psi_{k}$ that represent the Sun.

The cases in which the EO is shifted (panel c) or the coronagraph is shifted (panel d) are similar to each other. The umbra-penumbra pattern in the aperture plane becomes nonsymmetrical. We define a new axis (red dash-dotted line in Fig. 5) through the centers of the EO and the coronagraph. The new axis is tilted by the angle $\phi=\sqrt{\Delta x^{2}+\Delta y^{2}} / z_{0} \leq 10 \operatorname{arcsec}$ with respect to the original axis (black dash-dotted line) as the maximal expected shift is $\sqrt{\Delta x^{2}+\Delta y^{2}} \sim 7.0 \mathrm{~mm}$. In the new reference frame we can neglect the tilt of the EO and take into account the tilt of the coronagraph and shift of the Sun; the coaligned coronagraph is denoted by the red dashed line. Thus, these two cases can be considered as a simultaneous shift of the Sun and tilt of the coronagraph, where the two effects enhance each other.

In the case of the tilt of the coronagraph (panel e), the main effect is that in the $O^{\prime}$ plane the projections of the Sun and the EO are displaced with respect to the IO (see Fig. 7). Then more diffracted light propagates behind the IO on one side, and less diffracted light propagates behind the IO on the other side. Another effect is the inclination of the EO image (and thus its defocusing), but is less important because of small expected values of the tilt $\sim 25$ arcsec. Obviously, the overoccultation by the IO should be large enough to cover possible shifts of the EO image. The coronagraph and its mathematical model become nonsymmetrical: the two waves propagating from different directions $\boldsymbol{k}_{\rho \varphi_{1}}$ and $\boldsymbol{k}_{\rho \varphi_{2}}$ produce different responses in $D$. From the computational point of view, the tilt of the coronagraph around 
a) Symmetrical case

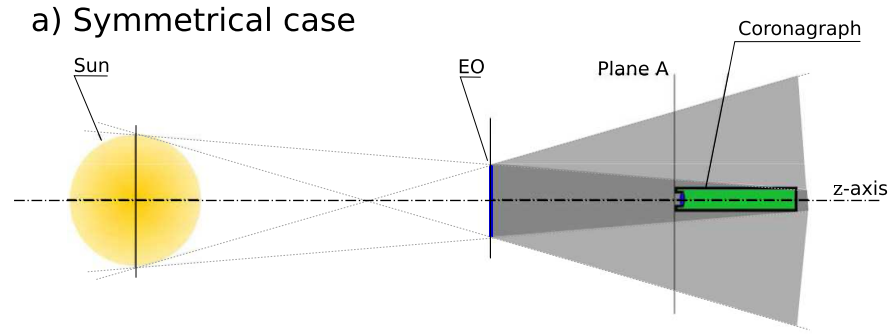

c) EO is shifted

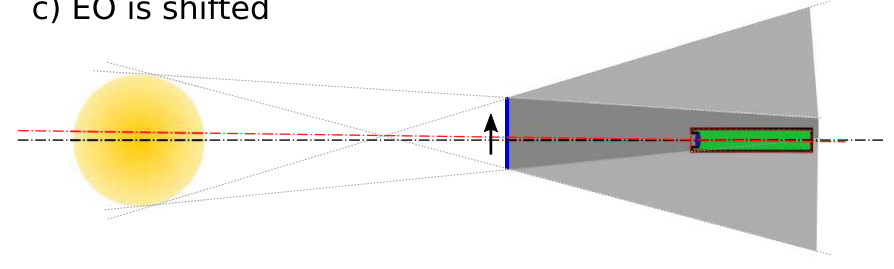

b) Sun is shifted

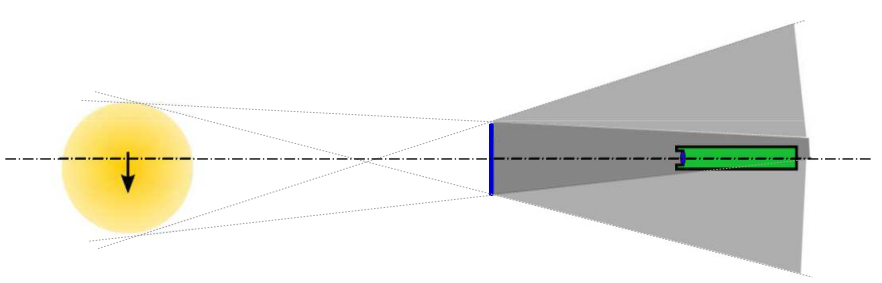

d) Coronagraph is shifted

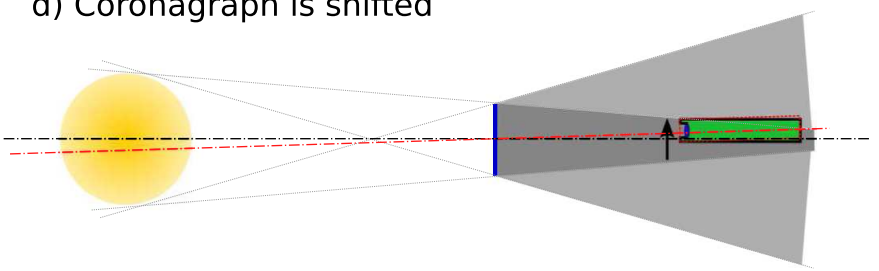

e) Coronagraph is tilted

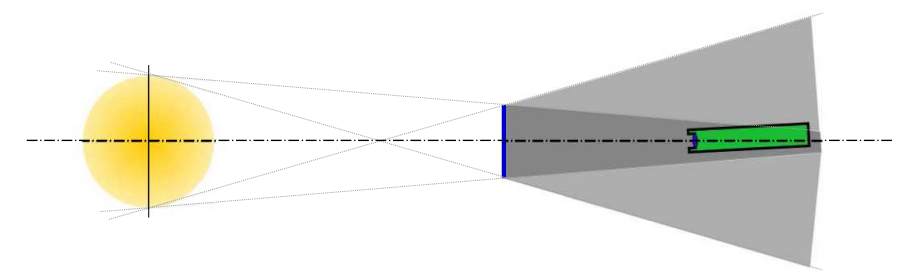

Fig. 5. Comparison of various misalignments. Panel $a$ : The symmetrical case is represented; panel $b$ : the shift of the Sun is shown; panel $c$ : the shift of the EO is depicted; panel $d$ : the shift of the coronagraph is depicted, and panel $e$ : the tilt of the coronagraph is represented. In panels $c$ and $d$ the red axis goes through centers of the EO and the entrance aperture, and dotted rectangles represent the coronagraph co-aligned with this new axis.
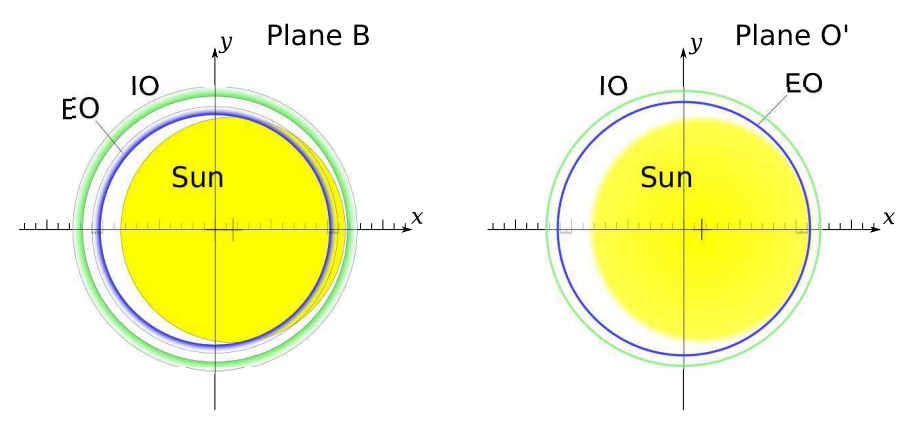

Fig. 6. Projections of the Sun, EO, and IO onto planes $B$ and $O^{\prime}$ in case of a shifted Sun (cf. Fig. 2).

the $O y$-axis is equivalent to multiplication of every $\Psi_{A k}$ by the complex factor $\exp \left(-2 \pi i \frac{\Theta x}{\lambda}\right)$.

Transversal displacement of the IO is very similar to the case of the tilt of the coronagraph, so we do not present additional computations for it. In the case of transversal displacement of the Lyot stop, the entrance aperture remains in the center of the umbra-penumbra pattern - the aperture receives minimal possible level of the diffracted light - and the bright diffraction ring is co-centered with the IO (i.e., the IO acts with maximal efficiency). Thus we conclude that the influence of this misalignment is smaller than that produced by the shift of the Sun and we do not consider it separately.
The case with the change of $z_{0}$ results in two effects: the change of level of the diffracted light on the entrance aperture, and change of the size of the diffraction ring in the $O^{\prime}$ plane. The combined impact of the two effects in the detector plane is not obvious and is analyzed below. From the computational point of view, the misalignment requires recalculation of the axisymmetrical function $\Psi_{A 00}$ mentioned in Sect. 2.3 (see also Appendix B).

The effect of the longitudinal displacement of the IO from the $O^{\prime}$ plane results in more diffracted light potentially propagate beyond the IO. From the computational point of view, it changes the $\Psi_{O^{\prime}}$ function (see Appendix A).

\section{Results of computations}

We consider $3 \sigma$ values of the misalignments, resulting from the FF accuracy (see Sect. 1): the relative shift of the Sun by $\phi=10$ arcsec owing to the transversal displacement of the satellites; the tilt of the coronagraph by $\Theta \sim 10$ arcsec, owing to the transversal displacement of the satellites, and 25 arcsec, owing to the tilt of the satellite plus the misalignment of the telescope; and the change of $z_{0}$ by $+15 \mathrm{~mm}$ and $-100 \mathrm{~mm}$ longitudinal displacement of the IO by $\Delta z_{\mathrm{IO}}= \pm 60 \mu \mathrm{m}$; we exaggerate the expected $\Delta z_{0}=-15 \mathrm{~mm}$ to the extreme ${ }^{1}$.

1 Since the ASPIICS project is in the middle of the preparation phase, these values are subject to changes. 


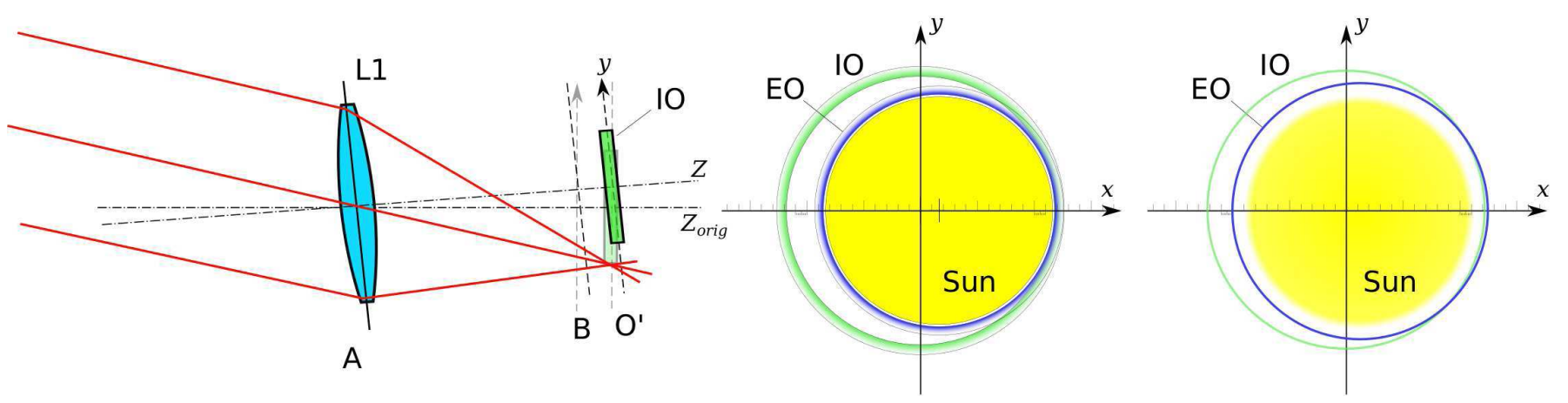

Fig. 7. Tilt of the coronagraph. Left panel: the relative displacement of the IO and the axes of the instruments are shown. Middle and right panels: the projections of the Sun, EO, and IO onto planes $B$ and $O^{\prime}$ are shown (cf. Figs. 2 and 6).

During the computations we used the parameters listed in Table 2 . We used $4 k \times 4 k$ arrays to represent $\Psi$ in each plane and sample the solar disk by $N=50$ points in the radial direction and by $M=400$ points in the polar direction (see Appendix $C$ for the analysis of the sampling).

We used the same solar limb darkening function as that used by RR17, which was initially proposed by van Hamme (1993), i.e.,

$B(\rho)=1-0.762\left(1-\sqrt{1-\rho^{2}}\right)-0.232\left(1-\rho^{2}\right) \log \left(\sqrt{1-\rho^{2}}\right)$,

where $\rho$ is the radial coordinate expressed as a fraction of the solar radius.

Since we are interested in the possibility of registering the signal by the ASPIICS detector, we converted the obtained brightness into number of photons pixel ${ }^{-1}$. For the conversion we used the following ASPIICS parameters (Renotte et al. 2016): aperture size $A=\pi R_{A}^{2}=19.6 \mathrm{~cm}^{2}$, angular size of a pixel of $2.8 \mathrm{arcsec}$, the exposure time $t_{\exp }=0.1 \mathrm{~s}$, and took the mean solar brightness as MSB $=2.08 \times 10^{20}$ photons $\mathrm{s}^{-1} \mathrm{~cm}^{-2} \mathrm{sr}^{-1}$ (the solar spectrum convolved with the spectral transmission of ASPIICS).

To compare the diffracted light with the corona, we took the K-corona brightness observed during solar maximum from Allen (1976). We take into account the geometrical vignetting, which is determined by the size of the IO. We applied linear vignetting function that starts (transmission $T=0 \%$ ) from the angle $v_{\min }=\arctan \left(\frac{R_{\mathrm{IO}}^{*}-R_{A}}{z_{0}}\right)$ and finishes $(T=100 \%)$ at the angle $v_{\text {max }}=\arctan \left(\frac{R_{\mathrm{IO}}^{*}+R_{A}}{z_{0}}\right)$, where $R_{\mathrm{IO}}^{*}$ stands for the size of the IO projected into $O$. In reality there is an additional effect at low angles. A high level of vignetting significantly widens the pointspread function of the coronagraph, which not only reduces the intensity, but also smears the image of the lower corona (see RR17 for the analysis of the effect).

Further on we show profiles of the signal in the $D$ plane taken in the direction where the increase of the diffracted light is maximal, i.e., in the $O x$ direction in $D$.

\subsection{Qualitative results}

Our numerical computations fully confirm the qualitative behavior of images in different planes as described in Sect. 3. In the $O^{\prime}$, plane the diffracted ring does not move because of solar shift, but it moves because of the tilt of the coronagraph. In the $D$ plane,

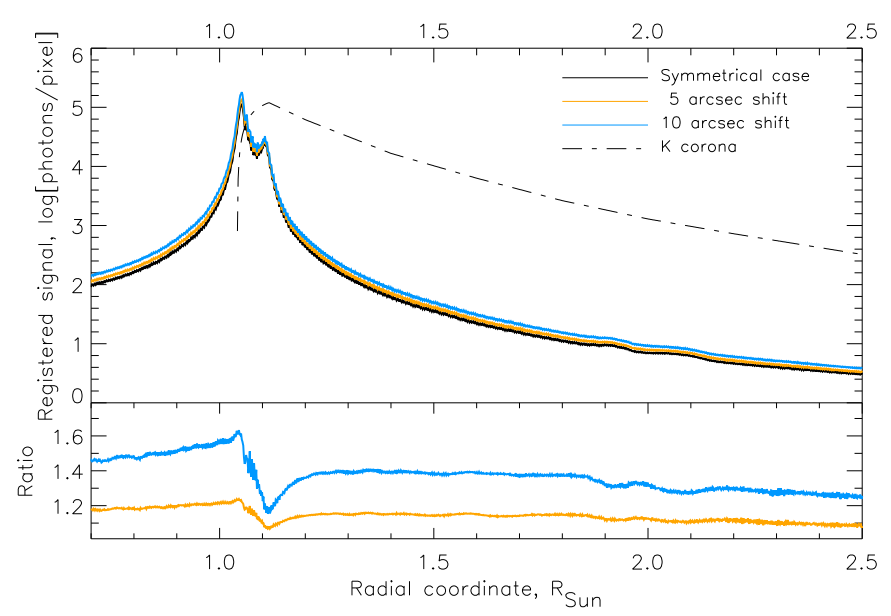

Fig. 8. Intensities of the diffracted light and corona for the case of the shift of the Sun. Top panel: radial profiles computed for the symmetrical and shifted cases are shown. The black curve denotes the symmetrical case, the yellow curve indicates the 5 arcsec shift, and the light blue curve indicates the 10 arcsec shift. The dash-dotted line represents the brightness of the K-corona. Bottom panel: ratios of shifted and symmetrical profiles of the diffracted light are shown.

the diffracted ring moves neither in the case of the solar shift nor in the case of the tilt of the coronagraph.

\subsection{Shift of the Sun and tilt of the coronagraph}

Intensities of the diffracted light for the case of the shift of the Sun are presented in Fig. 8. In the top panel the radial profiles correspond to different values of shift. The black curve indicates the symmetrical (unshifted) case, the yellow curve indicates $\phi=5$ arcsec, and the blue curve corresponds to $\phi=10$ arcsec. The dash-dotted line shows the brightness of the K-corona. In the bottom panel, ratios of shifted and symmetrical profiles are given.

Intensities of the diffracted light for the case of the tilt of the coronagraph are presented in Fig. 9. The black curve denotes the symmetrical case, the red curves indicates the shift $\Theta=10$ arcsec, the green curve indicates the $\Theta=25$ arcsec. The dash-dotted line shows the brightness of the K-corona. As mentioned above, the radial position of the diffracted profile maximum does not shift with the tilt of the coronagraph.

In Fig. 10 we present images in the plane $D$ computed for symmetrical, shifted, and tilted cases. All four panels are represented with the same quasi-logarithmic color table. 


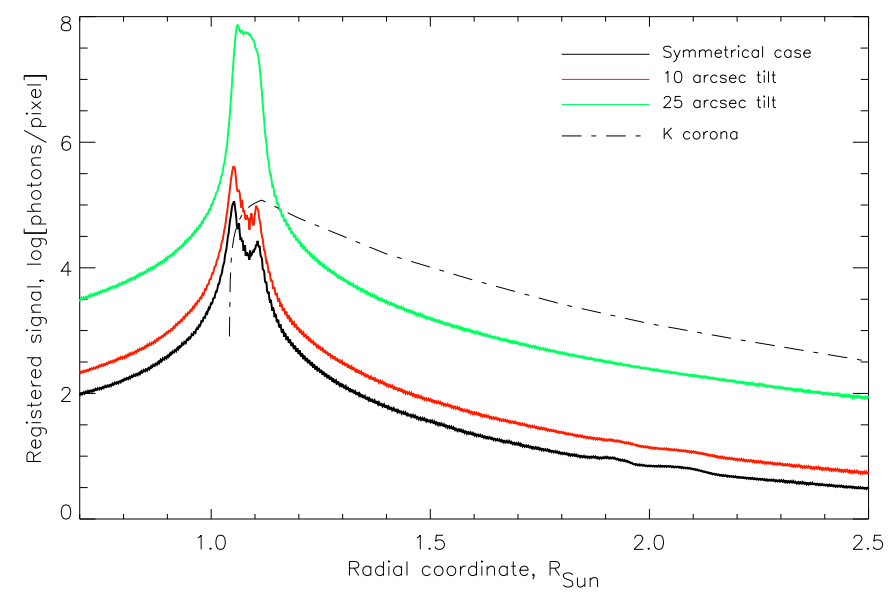

Fig. 9. Intensities of the diffracted light and corona for the case of the tilt of the coronagraph. The black curve denotes symmetrical case, the red curve indicates the 10 arcsec tilt, and the green curve indicates the 25 arcsec tilt. The dash-dotted line represents the brightness of the K-corona.

\subsection{Longitudinal displacement of the $1 O$ and change of the inter-satellite distance $z_{0}$}

The results for the relative displacements of the IO along the $z$-axis by $+60 \mu \mathrm{m}$ and $-60 \mu \mathrm{m}$; the results for the change of the inter-satellite distance $z_{0}$ by $+15 \mathrm{~mm}$ and $-100 \mathrm{~mm}$ are presented in Fig. 11. In the top panel, the radial profiles are given; in the bottom panel, the ratios of misaligned and symmetrical cases are given. All the changes are relatively small (up to 30\%) and can barely be seen in the top panel. While the result of reducing $z_{0}$ by $100 \mathrm{~mm}$ is pronounced in the bottom plot, the changes are invisible in the top panel. This is probably due to the oscillatory behavior of the obtained profile. The highest impact is produced by the longitudinal displacement of the IO by $+60 \mu \mathrm{m}$ further from L1, and the changes are comparable to those in the case of the shift of the Sun by 5 arcsec. The change of $z_{0}$ by $15 \mathrm{~mm}$ and the inward displacement of the IO almost does not change the overall intensity of the diffracted light.

\subsection{Choosing the proper 10 size}

It is clear that the major impact on the diffracted light is produced by the tilt of the coronagraph and by the misalignments that can be reduced to such a tilt. Already the tilt of $25 \operatorname{arcsec}$ is strong enough so that the diffracted light exceeds the level of the coronal intensity near the minimal heights. This is not unexpected if we consider projections of the EO and IO onto the $O^{\prime}$ plane. The rightmost edge of the $\mathrm{EO}$ has the coordinates $x_{\max }=z_{1} \tan \left(25^{\prime \prime}+\omega_{\mathrm{EO}}\right)=1.669 \mathrm{~mm}$, which is larger than $R_{\mathrm{IO}}=1.662 \mathrm{~mm}$. In other words, the bright diffraction ring is not fully blocked by the IO (this situation is shown in Fig. 7). Although the difference of $7 \mu \mathrm{m}$ is small, a lot of diffracted light propagates further in the instrument.

The impact of the tilt can be reduced if we increase the size of the IO. In Fig. 12 we compare the diffracted light for various sizes: $R_{\mathrm{IO}}=1.662 \mathrm{~mm}, 1.677 \mathrm{~mm}$, and $1.694 \mathrm{~mm}$ for the same tilt of 25 arcsec. Already $R_{\mathrm{IO}}=1.677 \mathrm{~mm}$ reduces the diffracted light by an order of magnitude. However, the diffracted light is still high enough at heights $<1.15 R_{\odot}$. The IO with $R_{\mathrm{IO}}=1.694 \mathrm{~mm}$ almost completely removes the effect of the tilt, as the intensity of the diffracted light becomes almost equal to the symmetrical case with $R_{\mathrm{IO}}=1.662 \mathrm{~mm}$. We note, however, that due to the increase of the IO both the peak of the diffracted light intensity and the coronal vignetting shift toward higher altitudes with respect to the symmetrical configuration. In the bottom part of Fig. 12 the vignetting functions corresponding to different IO sizes are given.

In order to choose the proper size of the IO, one has to take into account several misalignments simultaneously. An example of the combined effect of various misalignments is presented in Fig. 13. The internal occulter with $R_{\mathrm{IO}}=1.694 \mathrm{~mm}$ leads to the diffracted light below the coronal intensity in the unvignetted zone, even with all the misalignments combined. It is clear that the major impact is due to the tilt of the telescope.

\subsection{0 with apodized profiles}

Aime (2013) demonstrated that the use of apodized external occulters (i.e., those with variable transmission close to the edge) significantly decreases the level of diffraction light at the entrance aperture. Unfortunately, making an apodized external occulter for ASPIICS is a technical challenge. However, we demonstrate that apodizing the internal occulter may also lead to a significant (although quantitatively different) decrease of the diffracted light.

In Fig. 14 we compare the diffracted light for IOs with various apodized profiles, which have the linear gradient of widths 0.1 and $0.2 \mathrm{~mm}$, for the same tilt of 25 arcsec. The thick blue curve corresponds to the sharp-edge IO with $R_{\mathrm{IO}}=1.694 \mathrm{~mm}$. The thick red curve denotes the IO with $R \leq 1.662 \mathrm{~mm}$ opaque center and linear gradient $\Delta=0.1 \mathrm{~mm}$ apodization and the thick green curve denotes a similar IO but with $\Delta=0.2 \mathrm{~mm}$ apodization. The thin colored lines represent coronal signal with additional vignetting by IOs taken into account (colors correspond to the colors of diffraction curves), or the vignetting functions (given in the bottom part).

The IOs with apodization have superior performance with respect to larger sharp-edge IOs. The reduction of the diffracted light is significant. The profile of the IO with $\Delta=0.1 \mathrm{~mm}$ apodization and 25 arcsec tilt corresponds to the symmetrical case with $R_{\mathrm{IO}}=1.662 \mathrm{~mm}$ everywhere except for the innermost region of the corona. The IO with $\Delta=0.2 \mathrm{~mm}$ apodization has the intensity of the diffracted light in the inner region that is almost equal to the symmetrical case and has a significantly lower level (factor of 50) of diffracted light at heights $>1.1 R_{\odot}$.

Reduction of the coronal signal occurs in a small region within $1.25 R_{\odot}$ and the number of photons reaching the detector remains rather high (see thin curves in Fig. 14). For example, for the case of $\Delta=0.2 \mathrm{~mm}$ apodization and during $t_{\exp }=$ $0.1 \mathrm{~s}$ exposure time, the coronal signal remains at the average

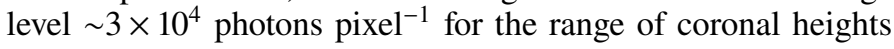
$1.1-1.25 R_{\odot}$. Such a photon flux allows reliable registration of the signal.

\section{Discussion}

\subsection{Comparison of various misalignments}

The performed computations show that the impact of various misalignments is considerably different for each type. For example, shifts of the EO and the coronagraph may have both severe and negligible impacts on the diffracted light. Nonsymmetrical umbra-penumbra pattern on the entrance aperture does not necessarily produce a severe impact (e.g., solar shift). Alternatively, a symmetrical umbra-penumbra pattern on the entrance aperture 


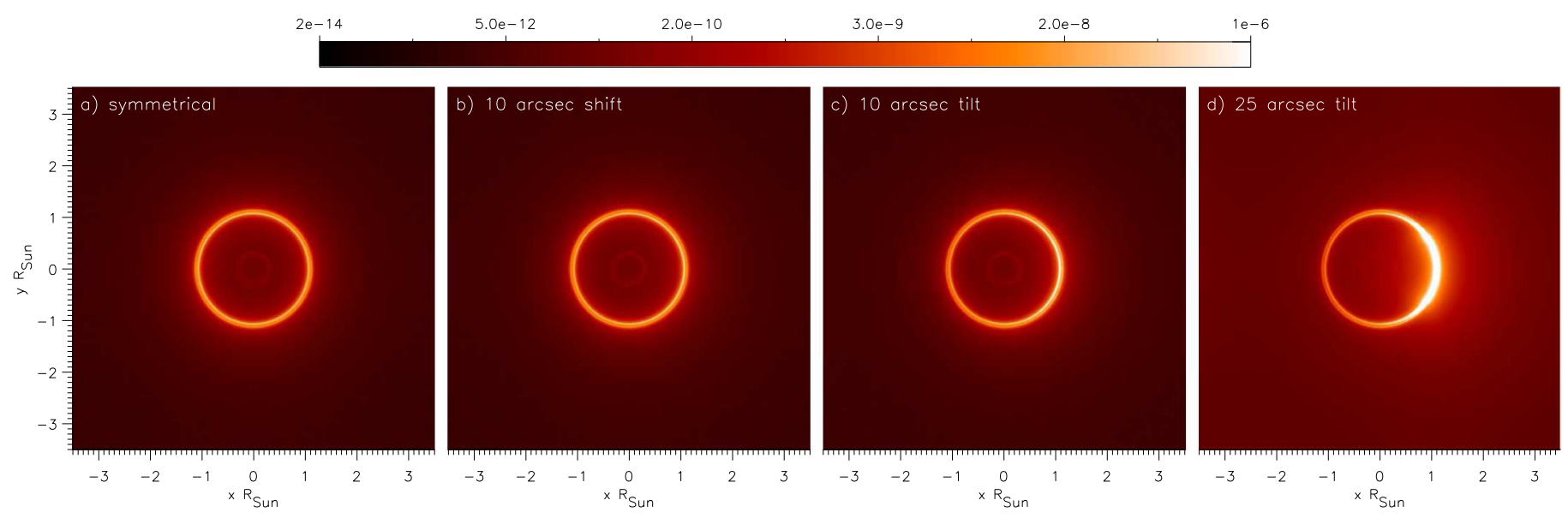

Fig. 10. Images in the plane $D$ computed for: panel $a$ : symmetrical case, panel $b$ : solar shift by 10 arcsec, panel $c$ : coronagraph tilt by 10 arcsec, and panel $d$ : coronagraph tilt by 25 arcsec. Color table is expressed in mean solar brightness.

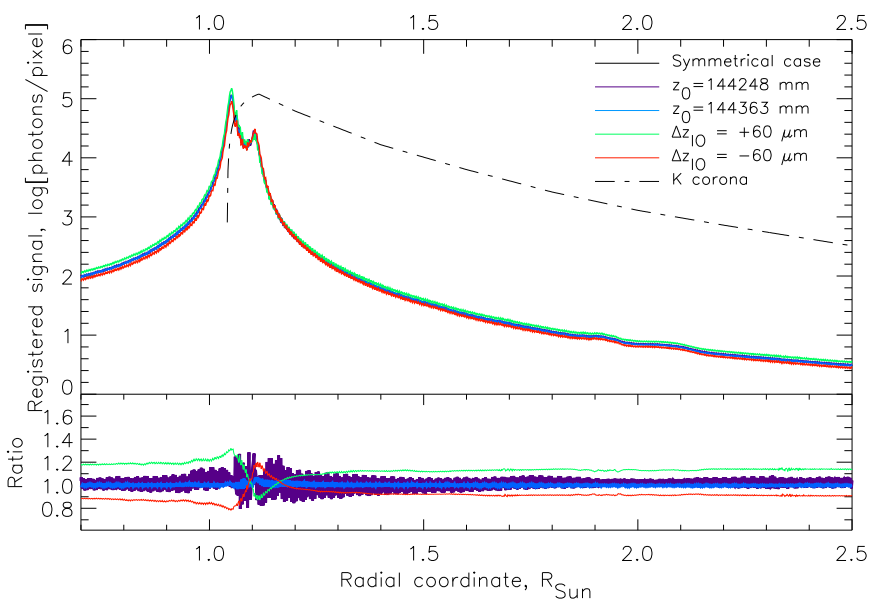

Fig. 11. Intensities of the diffracted light and corona for the case of the longitudinal displacement of the IO and change of $z_{0}$. Top panel: radial profiles of the diffracted light (colored curves) and the K-corona (dash-dotted line) are shown. Bottom panel: ratios of the displaced and symmetrical cases are shown.

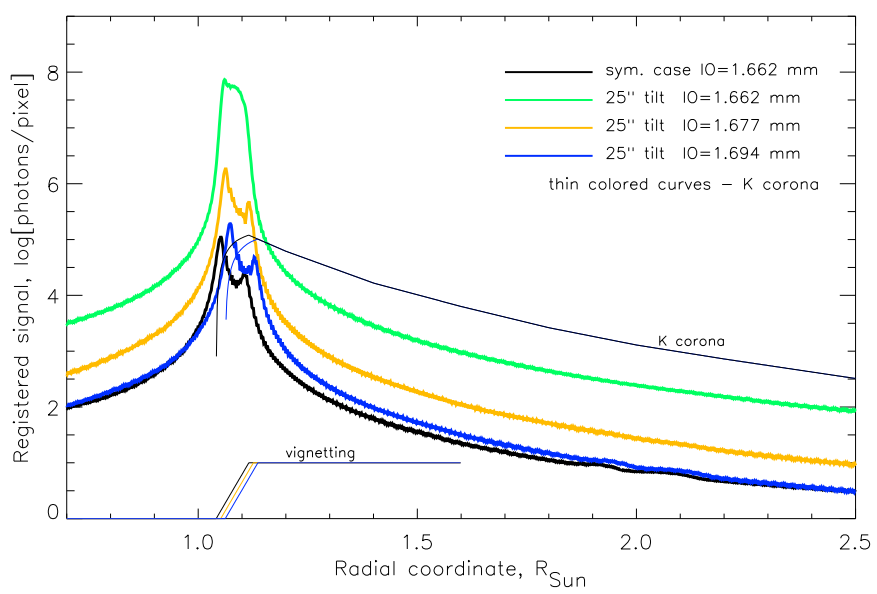

Fig. 12. Comparison of the diffracted light corresponding to different IO sizes for the same tilt of 25 arcsec. The green curve corresponds to $R_{\mathrm{IO}}=1.662 \mathrm{~mm}$, the yellow curve indicates $R_{\mathrm{IO}}=1.677 \mathrm{~mm}$, the blue curve indicates $R_{\mathrm{IO}}=1.694 \mathrm{~mm}$, and the black solid curve denotes symmetrical case with $R_{\mathrm{IO}}=1.662 \mathrm{~mm}$. Thin black and blue lines denote the coronal signal with vignetting taken into account or the vignetting functions (in the bottom part of the plot).

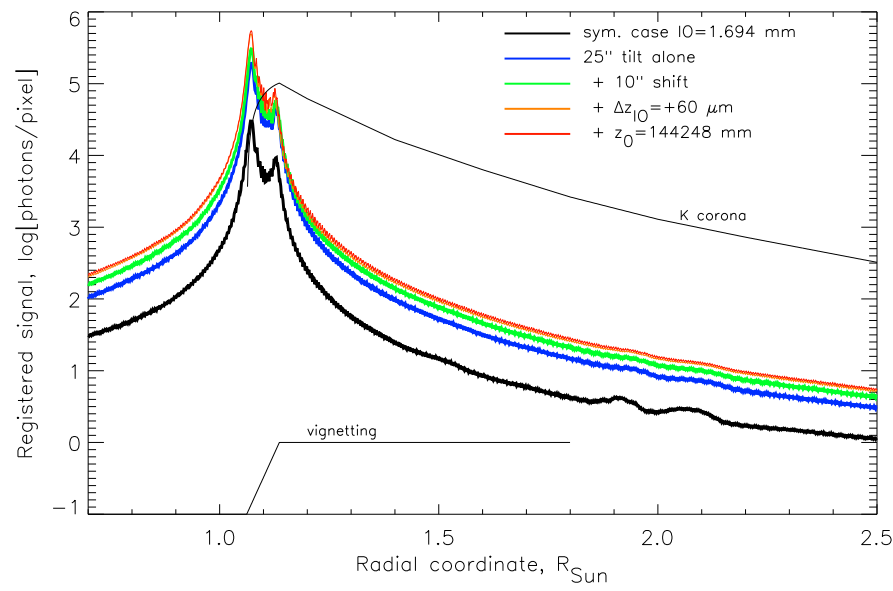

Fig. 13. Simultaneous effect of various misalignments for the IO with $R_{I O}=1.694 \mathrm{~mm}$. The black curve corresponds to the symmetrical case, the colored curves correspond to various combinations of misalignments: 25 arcsec tilt alone (blue); 25 arcsec tilt and 10 arcsec shift of the Sun (green); 25 arcsec tilt, 10 arcsec shift of the Sun and $60 \mu \mathrm{m}$ displacement of the internal occulter (orange); 25 arcsec tilt, 10 arcsec shift of the Sun, $60 \mu \mathrm{m}$ displacement of the internal occulter and $\Delta z_{0}=-100 \mathrm{~mm}$ change of the inter-satellite distance (red). The thin curve corresponds to the intensity of the K-corona taking into account the vignetting function shown in the bottom part of the plot.

does not guarantee good diffracted light performance (e.g., tilt of the coronagraph). Placing the EO either closer to the coronagraph or further away does not result in a significant increase of the diffracted light for the expected displacement. However, the displacement of the IO from its nominal position along the $z$-axis, which may resemble the displacement of the EO, either worsens or improves the performance depending on the position in the field of view.

\subsection{Using apodized IOs}

As we show in Sect. 5.5 that the IO with $0.2 \mathrm{~mm}$ apodization significantly reduces the level of diffracted light in tilted cases. Obviously, the performance of the apodized IO is even better at smaller tilts or in symmetrical cases.

The reduction of the coronal signal remains reasonable, as the inner corona is bright enough. Furthermore, the reduction of dynamic range produced by apodized IOs may further improve 


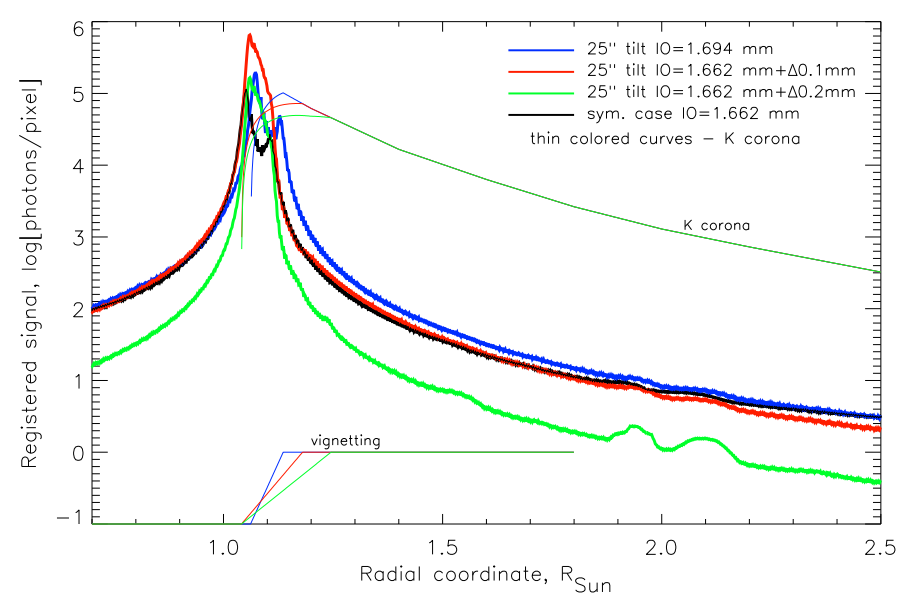

Fig. 14. Comparison of IOs with various apodization for the same tilt of 25 arcsec. The blue curve corresponds to sharp-edge IO with $R_{\mathrm{IO}}=1.694 \mathrm{~mm}$, the red curve indicates the $\mathrm{IO}$ with $R_{\mathrm{IO}}=1.662 \mathrm{~mm}$ opaque part and $\Delta=0.1 \mathrm{~mm}$ gradient, and the green curve indicates the $\Delta=0.2 \mathrm{~mm}$ gradient. The thin colored lines denote coronal signal with corresponding vignetting taken into account and vignetting functions (in the bottom part of the plot).

the performance of the coronagraph by diminishing the level of ghosts and scattered light in the instrument.

Using a smaller opaque region in apodized IOs potentially provides additional advantages because it reduces $v_{\min }-$ the height at which vignetting starts to disappear. In our case apodized IOs have $v_{\min }=1.078 R_{\odot}$, whereas a larger IO with $R=1.694 \mathrm{~mm}$ has $v_{\min }=1.099 R_{\odot}$. From this point view, even the IO with apodization $\Delta=0.1 \mathrm{~mm}$ may have an advantage over larger sharp-edge IOs with the same level of diffraction.

From a technological point of view, manufacturing of apodized IOs may be easier than that of sharp-edge IOs: producing a very sharp edge may not be possible and the remaining edge irregularities may additionally increase diffraction. In any case, accurate measurements of the IO geometry and transmission are of vital importance for processing of the registered coronal images.

Potential phase shifts of the wavefront due to propagation through the apodized IOs still need to be investigated.

\subsection{Dependence on the wavelength}

The ASPIICS coronagraph is equipped with a filter wheel with six positions that can be switched between individual exposures (Renotte et al. 2016). There are three passbands: wideband 535-565 nm, narrowband $530.4 \mathrm{~nm}$ for the Fe XIV line, and narrowband $587.7 \mathrm{~nm}$ for the He I line. Three additional positions are equipped with polarizers (rotated by $60^{\circ}$ with respect to each other) together with the wideband filter. There are various effects that influence the performance of ASPIICS in various passbands. First, chromatic aberrations of the primary objective and the rest of the optics modify the point-spread-function of the telescope very slightly. Second, it is essential that in the narrowband filters the observed coronal structures are bright in both spectral lines and continuum, thus the relative effect of the diffraction is less important.

For diffraction-related issues, we use the following reasoning. As a result of the property of diffraction discussed in Sect. 3, the diffraction image in the $O^{\prime}$ plane looks like a bright ring with the angular size of the EO. Obviously, this property is valid for any wavelength, despite the fact that the diffraction pattern from an individual plane-parallel wave $\Psi_{A k}$ depends on $\lambda$ and the diffraction at the entrance aperture also depends on $\lambda$. As a consequence, occulting the beam by the IO occurs with almost the same efficiency, regardless of the particular wavelength. Additional effects, such as different radial decrease of the intensity of the bright ring (the radial size of the Airy spot scales as $1.22 \lambda D / f$ ) may still be present, but the major effect of blocking the diffracted light by the IO is the same.

Thus we conclude that for multiwavelength observations diffraction effects do not change. A more detailed analysis of the spectral dependence of the diffraction will be carried out in the future.

\section{Conclusions}

We analyzed different types of misalignments in the externally occulted ASPIICS coronagraph on board the PROBA-3 mission, which has a very small overoccultation of the solar disk. We considered impacts of misalignments, from the physical point of view, and their computational realizations. We computed the resulting pattern of the diffracted light in the detector plane and compared this pattern with the coronal intensity.

Our computations of the diffracted light can be applied to any coronagraph with arbitrary geometry. However, for the case of ASPIICS we show the exceptional importance of precise coalignment. The most important misalignment is the tilt of the coronagraph. Special care should be taken to co-align the external and internal occulters. The true optical axis of the instrument (through the center of the IO and the entrance aperture) should be pointed to the center of EO as close as possible. The margin for the acceptable tilts $\sim 25$ arcsec is determined by oversizing the IO over the EO; the margin can be increased in the case of an apodized IO. The impacts of other misalignments are significantly smaller. Since the tilt of the coronagraph can be potentially corrected in flight by correcting the attitude of the coronagraph satellite, there is a possibility to reduce the size of the IO and, as a consequence, to reduce the minimal observed height of the corona.

We found that apodized IOs have very good diffracted light rejection performance, and are especially resistant to tilts.

Acknowledgements. We acknowledge support from the Belgian Federal Science Policy Office through the ESA - PRODEX programme (grant No. 4000117262). Authors are grateful to Dr. Anton Reva for his help during the preparation of the manuscript. We are grateful to the anonymous referee for numerous suggestions, which improved the paper.

\section{References}

Aime, C. 2007, A\&A, 467, 317

Aime, C. 2013, A\&A, 558, A138

Allen, C. W. 1976, Astrophysical Quantities, 3rd edn. (London: Athlon)

Bemporad, A., Baccani, C., Capobianco, G., et al. 2015, in Solar Physics and Space Weather Instrumentation VI, Proc. SPIE, 9604, 96040C

Berghmans, D., Hochedez, J. F., Defise, J. M., et al. 2006, Adv. Space Res., 38, 1807

Bout, M., Lamy, P., Maucherat, A., Colin, C., \& Llebaria, A. 2000, Appl. Opt., 39,3955

Brueckner, G. E., Howard, R. A., Koomen, M. J., \& Korendyke, C. M. 1995, Solar Phys., 162, 357

D’Huys, E., Seaton, D. B., De Groof, A., Berghmans, D., \& Poedts, S. 2017, J. Space Weather Space Clim., 7, A7

Fort, B., Morel, C., \& Spaak, G. 1978, A\&A, 63, 243

Frazin, R. A., Vásquez, A. M., Thompson, W. T., et al. 2012, Sol. Phys., 280, 273

Frigo, M., \& Johnson, S. G. 2005, Proc. IEEE, 93, 216

Galy, C., Fineschi, S., Galano, D., et al. 2015, in Solar Physics and Space Weather Instrumentation VI, Proc. SPIE, 9604, 96040B 
S. V. Shestov \& A. N. Zhukov: Misalignments in externally occulted solar coronagraphs

Goodman, J. 2005, McGraw-Hill physical and quantum electronics series, Introduction to Fourier Optics (W. H. Freeman)

Howard, R. A., Moses, J. D., Vourlidas, A., et al. 2008, Space Sci. Rev., 136, 67

Kim, I. S., Nasonova, L. P., Lisin, D. V., Popov, V. V., \& Krusanova, N. L. 2017, J. Geophys. Res.: Space Phys., 122, 77

Koutchmy, S. 1988, Space Sci. Rev., 47, 95

Krist, J. E. 2007, in Optical Modeling and Performance Predictions III, Proc. SPIE, 6675, 66750P

Kuzin, S. V., Zhitnik, I. A., Shestov, S. V., et al. 2011, Solar Syst. Res., 45, 162

Lamy, P., Damé, L., Vivès, S., \& Zhukov, A. 2010, in Space Telescopes and Instrumentation 2010: Optical, Infrared, and Millimeter Wave, Proc. SPIE, 7731,773118

Landini, F., Mazzoli, A., Venet, M., et al. 2010, in Ground-based and Airborne Instrumentation for Astronomy III, Proc. SPIE, 7735, 77354D
Lenskii, A. V. 1988, Sov. Astron., 25, 366

Renotte, E., Alia, A., Bemporad, A., et al. 2015, in Solar Physics and Space Weather Instrumentation VI, Proc. SPIE, 9604, 96040A

Renotte, E., Buckley, S., Cernica, I., et al. 2016, Proc. SPIE, 9904, 121

Reva, A. A., Ulyanov, A. S., Bogachev, S. A., \& Kuzin, S. V. 2014, ApJ, 793, 140

Rougeot, R., Flamary, R., Galano, D., \& Aime, C. 2017, A\&A, 599, A2

Seaton, D. B., Berghmans, D., Nicula, B., et al. 2013, Sol. Phys., 286, 43

Sirbu, D., Kim, Y., Kasdin, N. J., \& Vanderbei, R. J. 2016, Appl. Opt., 55, 6083

Slemzin, V., Bougaenko, O., Ignatiev, A., et al. 2008, Ann. Geophys., 26, 3007

van Hamme, W. 1993, AJ, 106, 2096

Venet, M., Bazin, C., Koutchmy, S., \& Lamy, P. 2010, in International Conference on Space Optics, Proc. SPIE, 10565, 105651W

Zhitnik, I., Kuzin, S., Afanas'ev, A., et al. 2003, Adv. Space Res., 32, 473

Zhukov, A. N., Saez, F., Lamy, P., Llebaria, A., \& Stenborg, G. 2008, ApJ, 680, 1532 


\section{Appendix A: Details of the mathematical approach}

In our analysis we followed the method of Aime (2013) and RR17. First we considered the propagation of a wave $\Psi$ through an optical system (Goodman 2005). After propagation through an aperture $A$, at the distance $z$ the wave amplitude $\Psi_{z}$ is calculated as

$\Psi_{z}(x, y)=\frac{\exp \left(\frac{i \pi\left(x^{2}+y^{2}\right)}{\lambda z}\right)}{i \lambda z} \cdot \mathcal{F}\left\{A \cdot \Psi(\xi, \eta) \cdot \exp \left(\frac{i \pi\left(\xi^{2}+\eta^{2}\right)}{\lambda z}\right)\right\}$,

where $(x, y)$ are coordinates in the observer plane, $\mathcal{F}$ is Fourier transform, $(\xi, \eta)$ are coordinates in the aperture plane, $\Psi(\xi, \eta)$ is the amplitude of the initial wave, $\lambda$ is the wavelength, $A=A(\xi, \eta)$ is the aperture function that denotes transparency at point $(\xi, \eta)$.

If one places a convergent lens with the focal distance $f$ immediately after the aperture, one must add an additional term $\exp \left(-\frac{i \pi\left(\xi^{2}+\eta^{2}\right)}{\lambda f}\right)$ inside the $\mathcal{F}$ argument. In the case in which $z$ and $f$ coincide, one can obtain a well-known expression for the focal plane of the lens, i.e.,

$\Psi_{z}(x, y)=\frac{\exp \left(\frac{i \pi\left(x^{2}+y^{2}\right)}{\lambda z}\right)}{i \lambda z} \cdot \mathcal{F}\{A \cdot \Psi(\xi, \eta)\}$.

Keeping this in mind, we write the expression for the field amplitude in $O^{\prime}$

$$
\begin{aligned}
\Psi_{O^{\prime}} & =\frac{\exp \left(\frac{i \pi r^{2}}{\lambda z_{1}}\right)}{i \lambda z_{1}} \cdot \mathcal{F}\left\{A_{A} \cdot \Psi_{A} \cdot \exp \left(\frac{i \pi r^{2}}{\lambda z_{1}}\right) \cdot \exp \left(-\frac{i \pi r^{2}}{\lambda f}\right)\right\} \\
& =\frac{\exp \left(\frac{i \pi r^{2}}{\lambda z_{1}}\right)}{i \lambda z_{1}} \cdot \mathcal{F}_{O^{\prime}},
\end{aligned}
$$

for the field amplitude in $C$

$$
\begin{aligned}
\Psi_{C} & =\frac{\exp \left(\frac{i \pi r^{2}}{\lambda d}\right)}{i \lambda d} \cdot \mathcal{F}\left\{A_{O^{\prime}} \cdot \Psi_{O^{\prime}} \cdot \exp \left(\frac{i \pi r^{2}}{\lambda d}\right) \cdot \exp \left(-\frac{i \pi r^{2}}{\lambda f_{L 2}}\right)\right\} \\
& =\frac{\exp \left(\frac{i \pi r^{2}}{\lambda d}\right)}{(i \lambda)^{2} z_{1} d} \cdot \mathcal{F}_{C},
\end{aligned}
$$

and finally for the field amplitude in $D$

$$
\begin{aligned}
\Psi_{D} & =\frac{\exp \left(\frac{i \pi r^{2}}{\lambda l}\right)}{i \lambda l} \cdot \mathcal{F}\left\{A_{C} \cdot \Psi_{C} \cdot \exp \left(\frac{i \pi r^{2}}{\lambda l}\right) \cdot \exp \left(-\frac{i \pi r^{2}}{\lambda f_{L 3}}\right)\right\} \\
& =\frac{\exp \left(\frac{i \pi r^{2}}{\lambda l}\right)}{(i \lambda)^{3} z_{1} d l} \cdot \mathcal{F}\left\{A_{C} \cdot \mathcal{F}_{C} \cdot \exp \left[\frac{i \pi r^{2}}{\lambda}\left(\frac{1}{d}+\frac{1}{l}-\frac{1}{f_{L 3}}\right)\right]\right\} .
\end{aligned}
$$

One can further simplify the expressions for the case of the optical layout corresponding to the initial design (i.e., taking into account $1 / z_{0}+1 / z_{1}=1 / f, d=z_{1}, f_{L 2}=z_{1} / 2, l=f$, and $f_{L 3}=$ $z_{1} / 2$ ), but for consideration of displaced optical components one should take specific values.

We note that arguments in each Fourier transform are taken at a corresponding plane and the coordinates and linear scales are different in each case. To perform numerical modeling of the Fourier propagation, we substituted $2 \mathrm{D}$ functions $\Psi_{A}, A_{A}, \Psi_{O^{\prime}}$, $A_{O^{\prime}}, \exp \left(\frac{i \pi r^{2}}{\lambda z_{1}}\right)$, etc. by corresponding 2D arrays.
Now we consider the diffraction of a plane-parallel wave on an infinitely thin circular occulter. The problem was considered by Aime (2013), who found that the amplitude can be expressed via the amplitude of the initially coaxial wave $\Psi_{A 00}$ (see his Eq. (5)),

$\Psi_{A \alpha \beta}(x, y)=T_{\alpha \beta}(x, y) \cdot \Gamma_{\alpha \beta}(x, y) \cdot \Psi_{A 00}\left(x+\alpha z_{0}, y+\beta z_{0}\right)$,

where $\Psi_{A 00}(\xi, \eta)$ is the amplitude of the initially coaxial wave, and

$T_{\alpha \beta}(x, y)=\exp \left(-2 \pi i \frac{\alpha x+\beta y}{\lambda}\right) \quad$ (Tilt),
$\Gamma_{\alpha \beta}(x, y)=\exp \left(-\pi i \frac{\left(\alpha^{2}+\beta^{2}\right) z_{0}}{\lambda}\right) \quad$ (Offset).

The value $\Psi_{A 00}(\xi, \eta)$ is calculated using the Fourier-Hankel transform

$$
\Psi_{A 00}(\xi, \eta)=1-\frac{\varphi_{z 0}(r)}{i \lambda z_{0}} \int_{0}^{R} 2 \pi \rho \exp \left(i \pi \frac{\rho^{2}}{\lambda z_{0}}\right) J_{0}\left(2 \pi \frac{r \rho}{\lambda z_{0}}\right) \mathrm{d} \rho,
$$

where $r=\sqrt{\xi^{2}+\eta^{2}}$ is the radial coordinate in the $A$ plane, $\varphi_{z 0}=\exp \left(i \pi r^{2} / \lambda z_{0}\right)$, and $J_{0}(r)$ is the Bessel function of the first kind. The function $\Psi_{A 00}$ is circularly symmetrical and is calculated initially as a 1D array.

\section{Appendix B: Numerical issues}

Calculation of $\Psi_{A 00}$ is computationally very expensive: computation of $\Psi_{A 00}$ with the spatial sampling of $3 \mu \mathrm{m}$ takes almost $10 \mathrm{~h}$ using IDL language on a PC with Intel Core i5 6200 processor. So we computed the function once for the nominal $z_{0}=144348 \mathrm{~mm}$, displaced $\Delta z_{0}=+15,-15 \mathrm{~mm}$, and saved these results into separate files; $\lambda=550 \mathrm{~nm}$ and $R_{\mathrm{EO}}=710 \mathrm{~mm}$ were used. Computation of $\Psi_{A k}$ from $\Psi_{A 00}$ takes 2-3 s, and computation of $\Psi_{O^{\prime}}, \Psi_{C}, \Psi_{D}$ takes $\sim 15 \mathrm{~s}$ for $4 k \times 4 k$ arrays. The difficulty is due to the necessity of high spatial sampling of the Sun: if we want to sample, for example, by $N=50$ points in $\rho$ and $M=400$ points in $\varphi$, then the total integration time is $\tau=20 N M \mathrm{~s}$, or $\sim 110 \mathrm{~h}$. Such a long time is not acceptable for performing a parametric study.

For the case of the perfect symmetry, RR17 used a method that resembles our approach described in Appendix C: $\left|\Psi_{D \boldsymbol{k}}\right|^{2}$ is calculated for $\varphi=0$ and after that the obtained image is blurred over $2 \pi$ in the polar coordinate (due to the circular symmetry of the source - the Sun). Since in the present analysis we are interested in nonsymmetrical configurations, a similar approach cannot be used for the full Sun.

In order to speed up the computations we developed an MPIFortran version of the full sampling model. Using of Fortran with fftw (Frigo \& Johnson 2005) library significantly increases the computation speed: three sequential FFT transforms now take only $\sim 5$ s. Furthermore, the algorithm can be easily parallelized: an individual process computes a given subset of incident waves and after that all the $I_{D}$ are summed. As a result, most of the computations reported here were carried out using full-sampling approach with $N=50$ and $M=400$ points. A single computation with 128 MPI processes on a cluster with Intel Xeon E5-2680 processors took on average around $20 \mathrm{~min}$. The necessity for parallelization on our own cluster did not allow us to use general purpose optical libraries like PROPER (Krist 2007) ${ }^{2}$.

2 Available freely from SourceForge: http://proper-library. sourceforge.net 


\section{Appendix C: Influence of spatial sampling}

We analyzed the influence of spatial sampling of the Sun and sizes of the arrays representing $\Psi_{A}, \Psi_{O^{\prime}}$, and $\Psi_{D}$ on our results.

Most of the images presented in this work were computed with sampling $M=400$ points in $\varphi$. Such a sampling reveals modulation in the final images along the perimeter of the bright annulus (imagine 400 images from Fig. 4d rotated and stacked together). In order to simulate a higher sampling in $M$, we used the following method: we substituted each image $I_{D}$ with an image blurred in polar coordinate $I_{D}^{\prime}=1 / W \sum_{0}^{W-1} \operatorname{rot}\left(I_{D}, \alpha\right)$, where $I_{D}$ is a low-sampled image, $\alpha \in[0 \ldots 2 \pi / 400)$ are equally spaced $W$ points, and rot is a procedure for rotation of an image $I_{D}$ by an angle $\alpha$. We applied this additional step every time in a separate IDL procedure. The obtained blurred images coincided with unrotated images $I_{D}$ computed with $M=1600$ sampling, which have no modulation in $\varphi$. The small step size of the initial sampling $2 \pi / 400$ results in obtained $I_{D}^{\prime}$ being smooth in the polar coordinate. Our method resembles the method used by RR17 for simplified computations of symmetrical configurations (see their Eq. (12) and corresponding description).

The difference between images with different sampling in $\rho$ (with $N=10, N=50, N=200$, and $N=1000$ points) could be barely noticed (Fig. C.1) so we used $N=50$ sampling for the analysis.

We also tried to use a Cartesian strategy to sample the solar disk, sampling points uniformly along the $x$ - and $y$-axes. We expected that the polar strategy (points are sampled uniformly along $\rho$ and $\varphi$ ) tends to overconcentrate points at low $\rho$ values and assigns these points low importance owing to small $\mathrm{d} S$. The Cartesian strategy with $\approx 20000$ points (the same number as in the polar case) provided smoother images in $D$, i.e., with a smaller but still noticeable modulation in $\varphi$. However, such a strategy does not allow us to apply additional image blurring, thus the advantage of the Cartesian strategy over the polar strategy disappears.

The change of the size of arrays from $4 k \times 4 k$ to $8 k \times 8 k$ also did not produce any significant change. However, increases in computation time and volume of the data were significant.

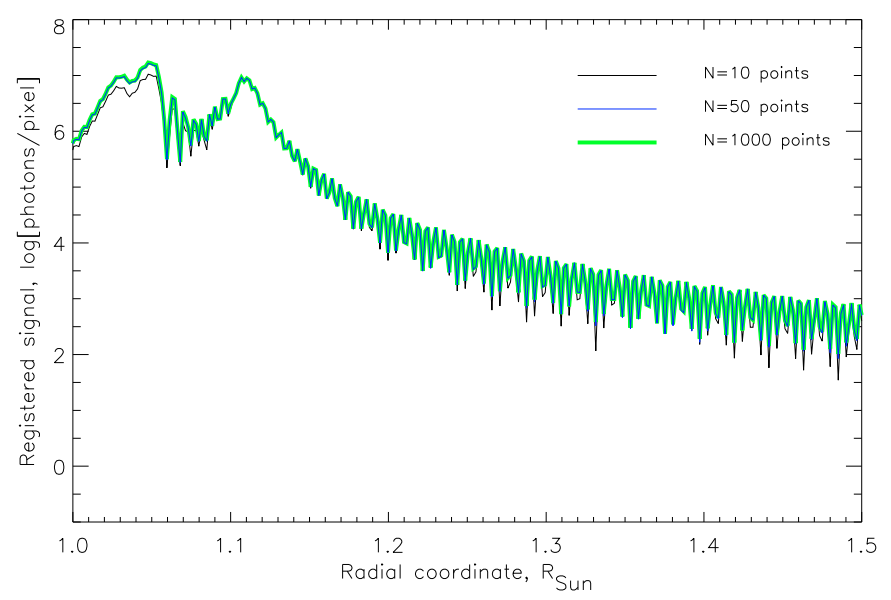

Fig. C.1. Comparison of various sampling of the Sun over the radius. The black curve corresponds to $N=10$ point, the thin blue curve corresponds to $N=50$ points, and thick green curve corresponds to $N=1000$ points.

The most significant influence on diffraction is the spatial sampling of the $\Psi_{A 00}$ function. Here we used $3 \mu \mathrm{m}$ spatial sampling, which is $\sim 6$ times smaller than $17 \mu \mathrm{m}$ the spatial scale in the $A$ plane (i.e., scale size of the $\Psi_{A}$ array). The reduction of $\Psi_{A 00}$ sampling to $7 \mu \mathrm{m}$ results in the development of ghost arches in the $D$ plane, whereas at $3 \mu \mathrm{m}$ these arches are almost invisible. The arches are weak even in case of the $7 \mu \mathrm{m}$ sampling and are most pronounced in the outer regions of the field of view, where the diffracted signal is low. An additional improvement of $\Psi_{A 00}$ sampling may further modify the level of the diffracted light in outer regions. However, we limit our analysis to $3 \mu \mathrm{m}$ sampling due to a level of diffraction at high altitudes that is already low and the presence of additional factors that may degrade the images (primarily ghost images produced by unwanted reflections from lens surfaces). 